\title{
Quantum statistical mechanics of gases in terms of dynamical filling fractions and scattering amplitudes
}

\author{
André LeClair \\ Newman Laboratory, Cornell University, Ithaca, NY
}

(Dated: 2006)

\begin{abstract}
We develop a finite temperature field theory formalism in any dimension that has the filling fractions as the basic dynamical variables. The formalism efficiently decouples zero temperature dynamics from the quantum statistical sums. The zero temperature 'data' is the scattering amplitudes. A saddle point condition leads to an integral equation which is similar in spirit to the thermodynamic Bethe ansatz for integrable models, and effectively resums infinite classes of diagrams. We present both relativistic and non-relativistic versions.
\end{abstract}




\section{INTRODUCTION}

For the investigation of finite temperature quantum field theory, the standard and welldeveloped formalism is based on the euclidean field theory with time compactified on a circle of circumference $\beta=1 / T$ where $T$ is the temperature. In practice, this involves the sums over Matsubara frequencies in perturbation theory [1, 2]. Though this formalism is very useful for some problems, such as the finite temperature dependence of the effective potential[3], for other properties, such as the equation of state involving the pressure and density, it would be desirable to have a formalism that more clearly preserves the classical picture of a gas of particles at given density and interacting via collisions.

It helps to realize that at least in principle it is possible to decouple the zero temperature dynamics and the quantum statistical sums. The argument is simple: the computation of the partition function $Z=\operatorname{Tr}\left(\mathrm{e}^{-\beta \mathrm{H}}\right)$ is in principle possible from the complete knowledge of the zero temperature eigenstates of the hamiltonian $H$. In practice this is rather difficult and one resorts to perturbative methods such as the Matsubara method, which unfortunately entangles the zero temperature dynamics from the quantum statistical mechanics. However there does exist a beautiful realization of this kind of decoupling for integrable quantum field theories in 2 spacetime dimensions due to Yang and Yang[4], which is refered to as the thermodynamic Bethe ansatz (TBA). (For the relativistic version, see [5].) In this formalism the decoupling is manifest: The free energy is expressed in terms of a pseudo-energy, in fact it takes the free field form, and the pseudo-energy is a solution of an integral equation whose kernel depends on the zero temperature S-matrix. In fact, the S-matrix and the free-particle dispersion relation are the only properties of the theory that are needed as input. The TBA is a very powerful tool for tracking RG flows and for computing the conformal central charge at the fixed points, and this was one of the main motivations for this work [25].

Dashen, Ma, and Bernstein derived an expression for the partition function in terms of the S-matrix in[6]. The derivation is very general, doesn't rely on integrability, and is valid in any number of spacetime dimensions[26]. The main result in [6] is rather formal, and a considerable amount of additional work is needed to churn it into a useful computational tool. Though some steps toward further developing the formalism were taken in [6] and [8], the program was never completed and appears to be eventually abandoned in favor of the Matsubara approach. As should be clear in our work, some important aspects of the 
approach were not well understood in the original works.

In this paper, we show that, with a new interpretation, the main formula of Dashen et. al., can serve as the starting point for a finite temperature formalism based on the physical occupation number densities and zero temperature scattering amplitudes. First, by using the cluster expansion for the S-matrix in a way not explointed in [6], we are able to resolve certain difficulties previously encountered and render the formalism considerably more appealing. Secondly, through a Legendre transformation we are able to formulate the quantum statistical mechanics directly in terms of dynamical filling fractions. By "dynamical" we refer to the property that they are determined from a variational principle, or saddle point condition, as in the TBA.

We wish to also point out the work of Lee and Yang on quantum statistical mechanics[9] which preceeded the TBA work of Yang and Yang. That approach is not based on the S-matrix but rather on matrix elements of $e^{-\beta H}$ itself. Since these matrix elements depend on the temperature, that approach does not disentangle the dynamics from the statistical sums. Nevertheless, we found some of the ideas, in particular diagramatic description, very useful.

An outline of the paper is the following. In the next section we describe in a completely general way how one formulates quantum statistical mechanics of gases in terms of dynamical filling fractions. In section III the main result of [6] is reviewed and some potential difficulties pointed out with so-called type B terms. In section IV, we show how the extensivity of the free energy follows from the cluster decomposition of the S-matrix, however in a delicate way that actually provides some constraints on the interpretation. We argue that the formalism is only consistent with the cluster decomposition if one passes to euclidean space. This also solves the problem with the type $\mathrm{B}$ terms. In section $\mathrm{V}$ we present a diagrammatic description of the formalism. It must be stressed that the resulting diagrams have nothing to do with finite temperature Matsubara/Feynman diagrams. In section VI we show that for the saddle point construction of section II, one needs only consider the 2-particle irreducible diagrams. The saddle point equation is an integral equation that automatically resums infinite numbers of diagrams. In section VII we consider contributions that come only from 2-body scattering. Here also the integral equation sums infinite numbers of 'foam' diagrams. Though we originally had in mind applications to relativistic field theory at high temperatures, our construction does not assume the underlying theory is Lorentz invariant. 
In section VIII we develop the non-relativistic case and solve the integral equation for a constant 2-body scattering amplitude.

In the concluding section we comment on the potential advantages of our formalism for certain classes of problems.

\section{FREE ENERGY AS A DYNAMICAL FUNCTIONAL OF FILLING FRAC- TIONS}

In the scattering description of quantum statistical mechanics that we will develop, a momentum space description is obviously appropriate. A very physical momentum space description uses the occupation number densities as the basic dynamical variables. In this section we generally describe how this can be done and illustrate it for free particles.

The free energy density (per volume) $\mathcal{F}$ is defined as

$$
\mathcal{F}=-\frac{1}{\beta V} \log Z, \quad Z=\operatorname{Tr} \mathrm{e}^{-\beta(\mathrm{H}-\mu \mathrm{N})}
$$

where $\beta=1 / T, \mu$ are the inverse temperature and chemical potential, $V$ is the d-dimensional spacial volume, and $H$ and $N$ are the hamiltonian and particle number operator. Since $\log Z$ is an extensive quantity, i.e. proportional to the volume, the pressure $p$ of the gas is minus the free energy density since $p=T \partial \log Z / \partial V=-\mathcal{F}$. For now, let us assume there is one species of bosonic $(s=1)$ or fermionic $(s=-1)$ particle. Given $\mathcal{F}(\mu)$, one can compute the thermally averaged number density $n$ :

$$
n=-\frac{\partial \mathcal{F}}{\partial \mu} \equiv \int \frac{d^{d} \mathbf{k}}{(2 \pi)^{d}} f(\mathbf{k})
$$

where $\mathbf{k}$ is the d-dimensional momentum vector. $(D=d+1$ is the spacetime dimension.) The dimensionless quantities $f$ are sometimes called the filling fractions.

One can express $\mathcal{F}$ as a functional of $f$ (and $\mu$ ) in a meaningful way with a Legendre transformation. Define

$$
G \equiv \mathcal{F}(\mu)+\mu n
$$

Treating $f$ and $\mu$ as independent variables, then using eq. (2) one has that $\partial_{\mu} G=0$ which implies it can be expressed only in terms of $f$ and satisfies $\delta G / \delta f=\mu$.

Inverting the above construction shows that there exists a functional $\digamma(f, \mu)$

$$
\digamma(f, \mu)=G(f)-\mu \int \frac{d^{d} \mathbf{k}}{(2 \pi)^{d}} f(\mathbf{k})
$$


which satisfies eq. (2) and is a stationary point with respect to $f$ :

$$
\frac{\delta \digamma}{\delta f}=0
$$

The above stationary condition is to be viewed as determining $f$ as a function of $\mu$. The

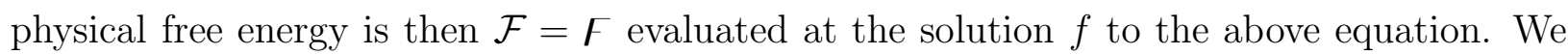
will refer to eq. (5) as the saddle point equation since it is suggestive of a saddle point approximation to a functional integral:

$$
Z=\int D f e^{-\beta V \digamma(f)} \approx e^{-\beta V \mathcal{F}}
$$

Let us illustrate these definitions for a free theory, in a way that will be useful in the sequel. In a free theory, the eigenstates of $H$ are multi-particle Fock space states $\left|\mathbf{k}_{1}, \mathbf{k}_{2} \ldots\right\rangle$. Let $\omega_{\mathbf{k}}$ denote the one-particle energy as a function of momentum $\mathbf{k}$. It is well-known that the trace over the multi-particle Fock space gives

$$
\mathcal{F}_{0}(\mu)=\frac{s}{\beta} \int \frac{d^{d} \mathbf{k}}{(2 \pi)^{d}} \log \left(1-s e^{-\beta\left(\omega_{\mathbf{k}}-\mu\right)}\right)
$$

From the definition eq. (2) one finds the filling fractions:

$$
f(\mathbf{k})=\frac{1}{e^{\beta\left(\omega_{\mathbf{k}}-\mu\right)}-s} \equiv f_{0}(\mathbf{k})
$$

In order to find the functional $\digamma(f, \mu)$ one first computes $G$ from eq. (3) and eliminates $\mu$ to express it in terms of $f$ using eq. (8). One finds

$$
\digamma_{0}(f, \mu)=\int \frac{d^{d} \mathbf{k}}{(2 \pi)^{d}}\left(\left(\omega_{\mathbf{k}}-\mu\right) f-\frac{1}{\beta}[(f+s) \log (1+s f)-f \log f]\right)
$$

One can then easily verify that $\delta \digamma / \delta f=0$ has the solution $f=f_{0}$ and plugging this back into eq. (9) gives the correct result eq. (17) for $\mathcal{F}_{0}$.

There is another way to view the above construction which involves the entropy. Write eq. (9) as

$$
\digamma=\mathcal{E}-\frac{1}{\beta} \mathcal{S}
$$

where $\mathcal{E}$ is the first $(\omega-\mu) f$ term in eq. (9), which is the energy density, and $\mathcal{S}$ is the remaining term in brackets. One can show by a standard counting argument, which involves the statistics of the particles, that $\mathcal{S}$ represents the entropy density of a gas of particles. (See for instance [10].) 
In the sequel, it will be convenient to trade the chemical potential variable $\mu$ for the variable $f_{0}$ :

$$
\digamma_{0}\left(f, f_{0}\right)=-\frac{1}{\beta} \int \frac{d^{d} \mathbf{k}}{(2 \pi)^{d}}\left(s \log (1+s f)+f \log \left(\frac{1+s f}{f} \frac{f_{0}}{1+s f_{0}}\right)\right)
$$

In section VI we will express the corrections to $\digamma$ for an interacting theory in terms of scattering amplitudes.

\section{FORMAL EXPRESSION FOR $Z$ IN TERMS OF THE S-MATRIX}

The trace that defines $Z$ is computed with respect to a complete set of orthogonal states $|\alpha\rangle:$

$$
\left\langle\alpha^{\prime} \mid \alpha\right\rangle=\delta_{\alpha^{\prime} \alpha}, \quad \mathbf{1}=\sum_{\alpha}|\alpha\rangle\langle\alpha| \quad \Longrightarrow Z=\sum_{\alpha}\left\langle\alpha\left|e^{-\beta(H-\mu N)}\right| \alpha\right\rangle
$$

Let us separate $H$ into free $\left(H_{0}\right)$ and interacting $\left(H_{1}\right)$ parts:

$$
H=H_{0}+H_{1}
$$

Since the states $\alpha$ in eq. (12) are not required to be eigenstates of $H$, let us take the trace over eigenstates of $H_{0}$ :

$$
H_{0}|\alpha\rangle=E_{\alpha}|\alpha\rangle
$$

In the next section we will specialize to plane-wave scattering states, but for the remainder of this section one need not be so specific.

It was shown how to express the thermal trace in terms of the S-matrix in [6]. The necessary algebraic tools are familiar from the formal theory of scattering [11, 12]. There is a simple derivation of what will turn out to be the essential term which goes as follows. For simplicity we first set the chemical potential $\mu$ to zero; it can easily be restored at the end by letting $\omega_{\mathbf{k}} \rightarrow \omega_{\mathbf{k}}-\mu$. Define the resolvent operator

$$
G(E)=\frac{1}{E-H+i \varepsilon}
$$

where $E$ is a real variable and $\varepsilon$ is small and positive. If $H$ is hermitian, then in the limit $\varepsilon \rightarrow 0^{+}:$

$$
G(E)-G(E)^{\dagger}=-2 \pi i \delta(E-H)
$$


Assuming that the spectrum of $H$ has $E \geq 0$, one then evidently has

$$
Z=-\frac{1}{2 \pi i} \int_{0}^{\infty} d E e^{-\beta E} \operatorname{Tr}\left(G(E)-G^{\dagger}(E)\right)
$$

Henceforth, we will not always display that operators depend on the variable $E$; all operators depend on $E$ except for $H, H_{0}, H_{1}$.

In order to obtain an expression that is meaningful when the trace is taken over the free Fock space, one wants to separate out expressions depending on $H_{0}$ as much as possible. The resolvent satisfies the equation[27]

$$
G=G_{0}+G_{0} H_{1} G
$$

where $G_{0}(E)=\left(E-H_{0}+i \varepsilon\right)^{-1}$. As in standard discussions of scattering (see e.g. [11]), define the operator

$$
T(E)=H_{1}+H_{1} G(E) H_{1}
$$

It satisfies

$$
H_{1} G=T G_{0}, \quad G H_{1}=G_{0} T
$$

Using eq. (20) one now has

$$
G=G_{0}+G_{0} T G_{0}
$$

and

$$
Z=Z_{0}-\frac{1}{\pi} \operatorname{Im} \int_{0}^{\infty} d E e^{-\beta E} \operatorname{Tr}\left(G_{0} T G_{0}\right)
$$

where $Z_{0}=\operatorname{Tr} e^{-\beta H_{0}}$ is the partition function of the free theory. Using now the cyclicity of the trace, $\partial_{E} G_{0}=-G_{0}^{2}$, an integration by parts, and $\operatorname{Im} G_{0}=-\pi \delta\left(E-H_{0}\right)$, one finds:

$$
Z=Z_{0}+Z_{A}+Z_{B}
$$

where

$$
Z_{A} \equiv-\beta \operatorname{Re}\left(\int d E e^{-\beta E} \operatorname{Tr}\left(\delta\left(\mathrm{E}-\mathrm{H}_{0}\right) \mathrm{T}(\mathrm{E})\right)\right)=-\beta \sum_{\alpha} e^{-\beta E_{\alpha}} \operatorname{Re}\left(T_{\alpha ; \alpha}\right)
$$

The term $Z_{B}$ involves $\partial_{E} T=-T G_{0}^{2} T$ and is thus quadratic in $T$. The derivation in [6] performs further algebraic manipulations of eq.(22) and elegantly expresses the final result for both $Z_{A, B}$ in terms of the S-matrix. The construction is summarized in Appendix A, where one finds:

$$
Z=Z_{0}+\frac{1}{4 \pi i} \int_{0}^{\infty} d E e^{-\beta E} \operatorname{Tr}\left(\mathrm{S}^{-1} \stackrel{\leftrightarrow}{\partial}_{\mathrm{E}} \mathrm{S}\right)
$$


where $X \stackrel{\leftrightarrow}{\partial}_{E} Y \equiv X\left(\partial_{E} Y\right)-\left(\partial_{E} X\right) Y$, and $S(E)$ is an operator valued function of $E$ related to the S-matrix [28]. More specifically,

$$
\begin{aligned}
S(E) & =1-2 \pi i \delta\left(E-H_{0}\right) T(E) \\
S^{-1}(E) & =1+2 \pi i \delta\left(E-H_{0}\right) T^{\dagger}(E)
\end{aligned}
$$

The on-shell matrix elements of $S(E)$ are the usual S-matrix elements:

$$
\left\langle\alpha^{\prime}|S(E)| \alpha\right\rangle=\left\langle\alpha^{\prime} \mid \alpha\right\rangle-2 \pi i \delta_{\alpha^{\prime} ; \alpha}^{(E)} T_{\alpha^{\prime} ; \alpha}, \quad \text { iff } \quad E=E_{\alpha}
$$

where $\delta_{\alpha^{\prime} ; \alpha}^{(E)} \equiv \delta\left(E_{\alpha^{\prime}}-E_{\alpha}\right)$. The condition "iff $E=E_{\alpha}$ " is what is referred to as "on-shell".

A significant amount of work remains in order to obtain a useful calculational tool from eq. (25), and this will be the main subject of the next section. For now, note that there are two types of terms (in addition to $Z_{0}$ ) in eq. (25) as in eq. (23), where

$$
\begin{aligned}
& Z_{A}=-\frac{1}{2} \int_{0}^{\infty} d E e^{-\beta E} \operatorname{Tr}\left[\partial_{\mathrm{E}}\left(\delta\left(\mathrm{E}-\mathrm{H}_{0}\right)\left(\mathrm{T}+\mathrm{T}^{\dagger}\right)\right)\right] \\
& Z_{B}=-i \pi \int_{0}^{\infty} d E e^{-\beta E} \operatorname{Tr}\left[\left(\delta\left(\mathrm{E}-\mathrm{H}_{0}\right) \mathrm{T}^{\dagger}\right) \stackrel{\leftrightarrow}{\partial}_{\mathrm{E}}\left(\delta\left(\mathrm{E}-\mathrm{H}_{0}\right) \mathrm{T}\right)\right]
\end{aligned}
$$

We will refer to these as Type $\mathrm{A}$ and Type $\mathrm{B}$ terms. Because of the $\delta\left(E-H_{0}\right)$ factors, we can now trace over eigenstates $|\alpha\rangle$ of $H_{0}$ and perform the integral over $E$. Consider first $Z_{A}$. Integrating by parts gives

$$
Z_{A}=-\beta \sum_{\alpha} e^{-\beta E_{\alpha}} \operatorname{Re}\left(T_{\alpha ; \alpha}\right)+\delta^{(E)}(0) \operatorname{Re}(\langle 0|T| 0\rangle)
$$

where $|0\rangle$ is the free-particle vacuum of zero energy. Assuming the vacuum is stable, $\langle 0|T| 0\rangle=0$ and the $\delta^{(E)}(0)$ term can be dropped and eq. (29) agrees with (24). $\left(\delta^{(E)}(0)\right.$ will be regularized below.)

The type A and B terms are quite different. The type B terms are actually rather peculiar since they may potentially spoil the extensivity of the free energy since they don't obviously have the same connectivity properties as the type A terms. We will return to this issue in the next section and actually propose that they should be discarded. $Z_{B}$ can be simplified using the optical theorem, which follows from $S^{-1} S=1$ :

$$
T-T^{\dagger}=-2 \pi i T^{\dagger} \delta\left(E-H_{0}\right) T
$$


Using this, and the cyclicity of the trace, one finds that the terms involving the derivative of the $\delta$-functions vanish. Inserting two complete sets of states one then finds:

$$
\begin{aligned}
Z_{B} & =-i \pi \sum_{\alpha, \alpha^{\prime}} e^{-\beta E_{\alpha}} \delta\left(E_{\alpha}-E_{\alpha^{\prime}}\right) T_{\alpha^{\prime} ; \alpha}^{*} \stackrel{\leftrightarrow}{\partial}_{E_{\alpha}} T_{\alpha^{\prime} ; \alpha} \\
& =2 \pi \sum_{\alpha, \alpha^{\prime}} e^{-\beta E_{\alpha}} \delta\left(E_{\alpha}-E_{\alpha^{\prime}}\right) \operatorname{Re}\left(T_{\alpha^{\prime} ; \alpha}\right) \stackrel{\leftrightarrow}{\partial}_{E_{\alpha}} \operatorname{Im}\left(T_{\alpha^{\prime} ; \alpha}\right)
\end{aligned}
$$

Note that the Type B terms vanish if $\operatorname{Im} T=0$.

\section{EXTENSIVITY OF THE FREE ENERGY AND THE CLUSTER DECOMPO- SITION}

In this section we specialize to a trace over plane wave scattering states and describe some new features that arise. In particular, in infinite volume, the free energy is expected to be extensive, i.e. proportional to the spacial volume $V$. So, one first must understand how all the various types of terms sum up in a way that can be reorganized as the exponential of something proportional to the volume. Clearly this has to do with properties of connectedness. In the present formalism, this property is essentially a consequence of the cluster decomposition of the S-matrix. The volume factors will arise from the following regularization of the momentum space delta-function:

$$
(2 \pi)^{d} \delta^{(d)}(0)=\lim _{\mathbf{k}=\mathbf{k}^{\prime}} \int d^{d} \mathbf{x} e^{i \mathbf{x} \cdot\left(\mathbf{k}-\mathbf{k}^{\prime}\right)} \equiv V
$$

If the expression (23) clusters in the expected way, then the free energy $-T \log Z$ can be identified with the sum of all terms with only one power of $V$. As we will show, requiring that the cluster decomposition for (23) leads to a free energy with this property actually provides some constraints on the interpretation of various terms.

\section{A. Fock space and S-matrix conventions}

Since we are considering a quantum field theory, the Hilbert space of the free theory is a Fock space. Let us now fix our normalizations for the free particle states and their scattering amplitudes. The creation-annihilation operators satisfy:

$$
a_{\mathbf{k}} a_{\mathbf{k}^{\prime}}^{\dagger}-s a_{\mathbf{k}^{\prime}}^{\dagger} a_{\mathbf{k}}=(2 \pi)^{d} \delta^{(d)}\left(\mathbf{k}-\mathbf{k}^{\prime}\right)
$$


The Hilbert space is then spanned by the multi-particle states

$$
\left|\mathbf{k}_{1} \mathbf{k}_{2} \cdots \mathbf{k}_{N}\right\rangle=\left(\prod_{i} \sqrt{2 \omega_{\mathbf{k}_{i}}}\right) a_{\mathbf{k}_{1}}^{\dagger} \cdots a_{\mathbf{k}_{N}}^{\dagger}|0\rangle
$$

satisfying

$$
H_{0}\left|\mathbf{k}_{1} \mathbf{k}_{2} \cdots \mathbf{k}_{N}\right\rangle=\left(\sum_{i} \omega_{\mathbf{k}_{i}}\right)\left|\mathbf{k}_{1} \mathbf{k}_{2} \cdots \mathbf{k}_{N}\right\rangle
$$

(The factors $\sqrt{2 \omega_{\mathbf{k}}}$ are a matter of convention.) One has the non-zero inner products:

$$
\left\langle\mathbf{k}_{1}^{\prime} \cdots \mathbf{k}_{N}^{\prime} \mid \mathbf{k}_{1} \cdots \mathbf{k}_{N}\right\rangle=(2 \pi)^{N d} \sum_{\mathcal{P}} s^{p} \prod_{i=1}^{N} 2 \omega_{\mathbf{k}_{i}} \delta\left(\mathbf{k}_{i}^{\prime}-\mathbf{k}_{i}\right)
$$

where the sum is over the $N$ ! permutations $\mathcal{P}$ of the order of the $\mathbf{k}_{i}^{\prime}$ and $p(\mathcal{P})$ is the degree of the permutation such that $p=0(p=1)$ if $\mathcal{P}$ involves an even (odd) number of pairwise permutations of particles. The above implies the following resolution of the identity:

$$
\mathbf{1}=\sum_{N=0}^{\infty} \frac{1}{N !} \int \underline{\underline{d \mathbf{k}_{1}}} \underline{\underline{\underline{\mathbf{k}_{2}}}} \cdots \underline{\underline{d \mathbf{k}_{N}}}\left|\mathbf{k}_{1} \cdots \mathbf{k}_{N}\right\rangle\left\langle\mathbf{k}_{1} \cdots \mathbf{k}_{N}\right|
$$

where for convenience we have defined the notation:

$$
\int \underline{\underline{d \mathbf{k}}} \equiv \int \frac{d^{d} \mathbf{k}}{(2 \pi)^{d}} \frac{1}{2 \omega_{\mathbf{k}}}
$$

(We have chosen our normalization of states so that the above integration measure over $\mathbf{k}$ is Lorentz invariant. We emphasize however that we are not assuming the theory to be Lorentz invariant; a non-relativistic case is worked out in section VIII.)

\section{B. Cluster decomposition}

In order to simplify the notation, the free particle states $\left|\mathbf{k}_{1}, \mathbf{k}_{2}, \ldots, \mathbf{k}_{n}\right\rangle$ will be denoted as $|\mathbf{1 2} \ldots \mathbf{n}\rangle$ and the S-matrix elements as

$$
\left\langle\mathbf{k}_{1}^{\prime}, \mathbf{k}_{2}^{\prime}, \ldots, \mathbf{k}_{n}^{\prime}|S| \mathbf{k}_{1}, \mathbf{k}_{2}, \ldots, \mathbf{k}_{m}\right\rangle=S_{\mathbf{1}^{\prime} \mathbf{2}^{\prime} \cdots \mathbf{n}^{\prime} ; \mathbf{1 2} \cdots \mathbf{m}}
$$

For the S-matrix the cluster decomposition is based on the physical requirement that particles that are causally separated cannot scatter (see for instance [12]). The cluster decomposition also ensures that the free energy only depends on connected S-matrix elements. The cluster decomposition may be expressed as follows:

$$
\left\langle\alpha^{\prime}|S| \alpha\right\rangle=\sum_{\text {partitions }} s^{p} S_{\alpha_{1}^{\prime} ; \alpha_{1}}^{c} S_{\alpha_{2}^{\prime} ; \alpha_{2}}^{c} S_{\alpha_{3}^{\prime} ; \alpha_{3}}^{c} \cdots
$$


where the sum is over partitions of the state $|\alpha\rangle$ into clusters $\left|\alpha_{1}\right\rangle,\left|\alpha_{2}\right\rangle, \ldots$. (The number of particles in $\left|\alpha_{i}\right\rangle$ and $\left\langle\alpha_{i}^{\prime}\right|$ is not necessarily the same.) The above formula essentially defines what is meant by the connected matrix elements $S^{c}$. The particles are assumed to be stable which implies

$$
\left\langle\mathbf{k}_{1}^{\prime}|S| \mathbf{k}_{1}\right\rangle=\left\langle\mathbf{k}_{1}^{\prime} \mid \mathbf{k}_{1}\right\rangle=(2 \pi)^{d} 2 \omega_{\mathbf{k}_{1}} \delta^{(d)}\left(\mathbf{k}_{1}^{\prime}-\mathbf{k}_{1}\right) \equiv \delta_{\mathbf{1}^{\prime} \mathbf{1}}^{(\mathbf{k})}
$$

It will be convenient to express the cluster decomposition in terms of $\widehat{T}$ defined by:

$$
S=1+i \widehat{T}
$$

The cluster decomposition for $\widehat{T}$ is then same as for that of $S$ but without the terms involving only delta functions which come from the ' 1 ' in $S=1+i \widehat{T}$. (One can show that these additional terms involving only delta functions are what give rise to $Z_{0}$.) The connected matrix elements $\widehat{T}^{c}$ are characterized as having only a single overall momentum and energy conserving delta function and cannot be factorized into functions of only a subset of the momenta. Below we will need $\widehat{T}^{c}$ in terms of the conventional scattering amplitudes $\mathcal{M}$ :

$$
\widehat{T}_{\alpha^{\prime} ; \alpha}^{c}=-2 \pi \delta_{\alpha^{\prime} ; \alpha}^{(E)} T_{\alpha^{\prime} ; \alpha}=(2 \pi)^{d+1} \delta_{\alpha^{\prime} ; \alpha}^{(E)} \delta^{(d)}\left(\mathbf{k}_{\alpha}-\mathbf{k}_{\alpha^{\prime}}\right) \mathcal{M}_{\alpha^{\prime} ; \alpha}
$$

where $E_{\alpha}, \mathbf{k}_{\alpha}$ are the total energy and momentum of the state $|\alpha\rangle$ and $\delta_{\alpha^{\prime} ; \alpha}^{(E)} \equiv \delta^{(E)}\left(E_{\alpha^{\prime}}-E_{\alpha}\right)$.

For $2 \rightarrow 2$ and $3 \rightarrow 3$ particles the cluster decomposition for $\widehat{T}$ then reads:

$$
\begin{aligned}
\widehat{T}_{\mathbf{1}^{\prime} \mathbf{2}^{\prime} ; \mathbf{1 2}} & =\widehat{T}_{\mathbf{1}^{\prime} \mathbf{2}^{\prime} ; \mathbf{1 2}}^{c} \\
\widehat{T}_{\mathbf{1}^{\prime} \mathbf{2}^{\prime} \mathbf{3}^{\prime} ; \mathbf{1 2 3}} & =\widehat{T}_{\mathbf{1}^{\prime} \mathbf{2}^{\prime} \mathbf{3}^{\prime} ; \mathbf{1 2 3}}^{c}+\left[\delta_{\mathbf{1}^{\prime} \mathbf{1}}^{(\mathbf{k})} \widehat{T}_{\mathbf{2}^{\prime} \mathbf{3}^{\prime} ; \mathbf{2 3}}^{c} \pm \text { perm. }\right]_{9}
\end{aligned}
$$

The subscript 9 indicates the number of permutations within the brace. If the one-particle states were not stable, there would be additional terms. In order to illustrate some important additional features, we will also need the $4 \rightarrow 4$ particle decomposition:

$$
\begin{aligned}
\widehat{T}_{\mathbf{1}^{\prime} \mathbf{2}^{\prime} \mathbf{3}^{\prime} \mathbf{4}^{\prime} ; \mathbf{1 2 3 4}=\widehat{T}_{\mathbf{1}^{\prime} \mathbf{2}^{\prime} \mathbf{3}^{\prime} \mathbf{4}^{\prime} ; \mathbf{1 2 3 4}}^{c}+} & {\left[\widehat{T}_{\mathbf{1}^{\prime} \mathbf{2}^{\prime} ; \mathbf{1 2}}^{c} \widehat{T}_{\mathbf{3}^{\prime} \mathbf{4}^{\prime} ; \mathbf{3 4}}^{c} \pm \text { perm. }\right]_{18}+\left[\delta_{\mathbf{1}^{\prime} \mathbf{1}}^{(\mathbf{k})} \widehat{T}_{\mathbf{2}^{\prime} \mathbf{3}^{\prime} \mathbf{4}^{\prime} ; \mathbf{2 3 4}}^{c} \pm \text { perm. }\right]_{16} } \\
+ & {\left[\delta_{\mathbf{1}^{\prime} \mathbf{1}}^{(\mathbf{k})} \delta_{\mathbf{2}^{\prime} \mathbf{2}}^{(\mathbf{k})} \widehat{T}_{\mathbf{3}^{\prime} \mathbf{4}^{\prime} ; \mathbf{3 4}}^{c} \pm \text { perm. }\right]_{72} }
\end{aligned}
$$

\section{One-particle resummation and emergence of filling fractions}

To compute the required trace, one must set $\left\{\mathbf{k}^{\prime}=\mathbf{k}\right\}$. This leads to a more specialized cluster decomposition that is suitable to compute $Z$. For instance, the 9 terms in braces 
in eq. (44) separate into $3+6$ distinct types of terms depending on whether they contain $\delta^{(d)}(0)$ :

$$
\widehat{T}_{\mathbf{1 2 3} ; \mathbf{1 2 3}}=\widehat{T}_{\mathbf{1 2 3}, \mathbf{1 2 3}}^{c}+\left[(2 \pi)^{d} \delta^{(d)}(0) 2 \omega_{\mathbf{k}_{1}} \widehat{T}_{\mathbf{2 3} ; \mathbf{2 3}}^{c} \pm \text { perm. }\right]_{3}+\left[s \delta_{\mathbf{1 2}}^{(\mathbf{k})} \widehat{T}_{\mathbf{2 3} ; \mathbf{1 3}}^{c} \pm \text { perm. }\right]_{6}
$$

In order to illustrate the variety of terms that can arise from the cluster decomposition, let us compute the Type A terms for low numbers of particles. For simplicity we set the chemical potential to zero. For two particles one finds:

$$
\left.Z_{A}\right|_{2 \text { part. }}=\frac{\beta V}{2} \int \underline{\underline{d \mathbf{k}_{1}}} \underline{\underline{d \mathbf{k}_{2}}} e^{-\beta\left(\omega_{1}+\omega_{2}\right)} \operatorname{Re}\left(\mathcal{M}_{\mathbf{1 2} ; \mathbf{1 2}}\right)
$$

where we have used eq. (32). $\left(\omega_{i} \equiv \omega_{\mathbf{k}_{i}}\right)$

For 3 particles one finds

$$
\begin{aligned}
\left.Z_{A}\right|_{3 \text { part. }}=\frac{\beta V}{3 !} & \int \underline{\underline{d \mathbf{k}_{1}}} \underline{\underline{d \mathbf{k}_{2}}} \underline{\underline{d \mathbf{k}_{3}}} e^{-\beta\left(\omega_{1}+\omega_{2}+\omega_{3}\right)} \operatorname{Re}\left(\mathcal{M}_{\mathbf{1 2 3} ; \mathbf{1 2 3}}\right) \\
+ & s \frac{\beta V}{2} \int \underline{\underline{\underline{\mathbf{k}_{1}}}} \underline{\underline{\underline{\mathbf{k}_{2}}}} e^{-\beta\left(\omega_{1}+\omega_{2}\right)}\left(e^{-\beta \omega_{1}}+e^{-\beta \omega_{2}}\right) \operatorname{Re}\left(\mathcal{M}_{\mathbf{1 2} ; \mathbf{1 2}}\right) \\
& +\beta V^{2}\left(\int \underline{\underline{d \mathbf{k}_{1}}} \underline{\underline{\underline{\mathbf{k}_{2}}}} e^{-\beta\left(\omega_{1}+\omega_{2}\right)} \operatorname{Re}\left(\mathcal{M}_{\mathbf{1 2} ; \mathbf{1 2}}\right)\right)\left(\int \frac{d^{d} \mathbf{k}}{(2 \pi)^{d}} e^{-\beta \omega_{\mathbf{k}}}\right)(50
\end{aligned}
$$

These terms have the following interpretation. Eq. (48) is a new contribution of the same kind as eq. (47). The term (49) just modifies the integration measure $\underline{\underline{d \mathbf{k}}}$ for the (47) term. The combined measure factors are the first terms in the expansion of the free filling fraction $f_{0}$ defined in eq. (8). This manner in which the filling fraction emerges was also a feature of one approach to finite temperature correlation functions in [14, 15]. The last term is proportional to $V^{2}$ and is thus expected to be a $V^{2}$ term in the expression $Z=\exp (-\beta V \mathcal{F})$. One can easily verify that it is the correct combinatorial product of a term from the free contribution $\mathcal{F}_{0}$ and another from the two-particle contribution in eq. (47).

A simple combinatorial argument shows that one can sum up all the terms that are a product of one $\widehat{T}^{c}$ and some $\delta_{\mathbf{n m}}^{(\mathbf{k})}$ factors to obtain

$$
\mathcal{F}=\mathcal{F}_{0}-\sum_{N=2}^{\infty} \frac{1}{N !} \int\left(\prod_{n=1}^{N} \frac{d^{d} \mathbf{k}_{n}}{(2 \pi)^{d}} \frac{f_{0}\left(\mathbf{k}_{n}\right)}{2 \omega_{\mathbf{k}_{n}}}\right) \operatorname{Re}\left(\mathcal{M}_{\mathbf{1 2 . . N} ; \mathbf{1 2 . . . N}}\right)+\ldots . .
$$

Restoring the chemical potential $\mu$, the dependence on it is only through $f_{0}$ as given in eq. (8).

For a scalar field interacting with potential $V(\phi)=\lambda \phi^{4} / 4$ !, to lowest order $\mathcal{M}_{\mathbf{1 2} ; \mathbf{1 2}}=$ $-\lambda[13]$ and the 2-particle contribution in eq. (151) gives the same result to order $\lambda$, as the 
Matsubara approach[1]. (In the latter approach this arises as a two-loop finite temperature Feynman diagram.) The $3 \rightarrow 3$ particle contribution to eq. (51) was also shown to agree with the 3-loop result in [16].

\section{The need to continue to euclidean space}

There are additional contributions to $\mathcal{F}$ that come from terms in the cluster expansion that involve more than one $\widehat{T}^{c}$ factor. They first arise at four particles. The terms in $\widehat{T}_{1234 ; 1234}$ that give new contributions to $\mathcal{F}$ not already included in eq. (51) are the following:

$$
\widehat{T}_{\mathbf{1 2 3 4 ; 1 2 3 4}}=i\left[\widehat{T}_{\mathbf{1 2 ; 1 2}}^{c} \widehat{T}_{\mathbf{3 4} ; \mathbf{3 4}}^{c}\right]_{3}+i\left[s \widehat{T}_{\mathbf{1 3 ; 1 2}}^{c} \widehat{T}_{\mathbf{2 4} ; \mathbf{3 4}}^{c}\right]_{12}+i\left[\widehat{T}_{\mathbf{1 2 ; 3 4}}^{c} \widehat{T}_{\mathbf{3 4} ; \mathbf{1 2}}^{c}\right]_{3}+\ldots
$$

In order to compute their contribution to $Z_{A}$, we start with the expression (24) in terms of $T$.

We then obtain the cluster decomposition of $T$ from eq. (52) using $\widehat{T}_{\alpha^{\prime} ; \alpha}=-2 \pi \delta_{\alpha^{\prime} ; \alpha}^{(E)} T_{\alpha^{\prime} ; \alpha}$. In carrying this out, one sees that one can divide both sides of eq. (52) by $\delta^{(E)}(0)$ and obtain something well-defined. Finally we express the final result in terms of the scattering amplitudes $\mathcal{M}$. The first term in eq. (52) in this way leads to:

$$
\left.Z_{A}\right|_{4 \text { part. }}=\ldots . . \frac{\beta V^{2}}{8} R e\left[2 \pi i \delta^{(E)}(0)\left(\int \underline{\underline{d \mathbf{k}_{1}}} \underline{\underline{d \mathbf{k}_{2}}} e^{-\beta\left(\omega_{1}+\omega_{2}\right)} \mathcal{M}_{\mathbf{1 2 ; 1 2}}\right)^{2}\right]+\ldots . .
$$

Since this is proportional to $V^{2}$ it must come from the square of the 2-particle term in the expansion of $Z=(1+\ldots) \exp \left(\beta V / 2 \int \operatorname{Re}\left(\mathcal{M}_{\mathbf{1 2} ; \mathbf{1 2}}\right)\right)$. In attempting to match this with eq. (53), one notices a very interesting phenomenon: the two terms can only be identified after one makes the Wick rotation

$$
\delta_{\alpha^{\prime} ; \alpha}^{(E)} \rightarrow-i \delta_{\alpha^{\prime} ; \alpha}^{(E)}
$$

One then regularizes the $\delta^{(E)}(0)$ as follows:

$$
2 \pi \delta^{(E)}(0) \equiv \beta
$$

The above equation can be understood as following from the fact that in the Matsubara approach to finite temperature field theory, one goes to euclidean space and compactifies the time on a circle of circumference, i.e. volume, $\beta$. The above equation is then simply the euclidean time version of eq. (32).

Implementing the rules discussed in the last paragraph, the two additional sets of terms in the cluster decomposition (52) lead to two new contributions to the free energy. In the 
resummation of 1-particle terms, the filling fractions $f_{0}$ again emerge. These lead to:

$$
\begin{aligned}
\mathcal{F}=- & \frac{s \beta}{2} \int \frac{d^{d} \mathbf{k}_{1}}{(2 \pi)^{d}} \frac{d^{d} \mathbf{k}_{2}}{(2 \pi)^{d}} \frac{d^{d} \mathbf{k}_{3}}{(2 \pi)^{d}} \frac{f_{0}\left(\mathbf{k}_{1}\right)}{2 \omega_{1}} \frac{f_{0}\left(\mathbf{k}_{2}\right)^{2}}{4 \omega_{2}^{2}} \frac{f_{0}\left(\mathbf{k}_{3}\right)}{2 \omega_{3}} \operatorname{Re}\left(\mathcal{M}_{\mathbf{1 2} ; \mathbf{1 2}} \mathcal{M}_{\mathbf{2 3} ; \mathbf{2 3}}\right) \\
& -\frac{1}{8} \int \frac{d^{d} \mathbf{k}_{1}}{(2 \pi)^{d}} \frac{d^{d} \mathbf{k}_{2}}{(2 \pi)^{d}} \frac{d^{d} \mathbf{k}_{3}}{(2 \pi)^{d}}\left(\prod_{n=1}^{4} \frac{f_{0}\left(\mathbf{k}_{n}\right)}{2 \omega_{n}}\right) 2 \pi \delta_{12 ; 34}^{(E)} \operatorname{Re}\left(\mathcal{M}_{\mathbf{1 2} ; \mathbf{3 4}} \mathcal{M}_{\mathbf{3 4} ; \mathbf{1 2}}\right)+\ldots
\end{aligned}
$$

where $\mathbf{k}_{4}=\mathbf{k}_{1}+\mathbf{k}_{2}-\mathbf{k}_{3}$ and $\omega_{4}=\omega_{\mathbf{k}_{4}}$.

\section{E. $Z_{B}$ terms: To $\mathbf{B}$ or not to $\mathbf{B}$ ?}

We now return to issue of the type B terms in eq. (31). It is clear from the previous results of this section that the property of the extensivity of the free energy arises in a delicate way from the cluster decomposition. Since $Z=Z_{0}+Z_{A}+Z_{B}$ and the $Z_{B}$ terms are quadratic in $T$ they could very easily spoil the extensivity. We have already shown how $Z_{0}+Z_{A}$ exponentiates to something proportional to the volume, but the way this comes about is quite subtle.

We now argue that the need to go to euclidean space cures this potential problem in a very satisfying way. In trying to perform the match discussed in the last subsection, one actually needs a stronger constraint:

$$
\beta\left(\int \operatorname{Re}\left(\mathcal{M}_{\mathbf{1 2} ; \mathbf{1 2}}\right)\right)^{2}=\operatorname{Re}\left[2 \pi \delta^{(E)}(0)\left(\int \mathcal{M}_{\mathbf{1 2} ; \mathbf{1 2}}\right)^{2}\right]
$$

I.e. $\mathcal{M}_{\mathbf{1 2 ; 1 2}}$ must be real. It is well-known that the imaginary parts of scattering amplitudes arise through cuts when one is in Minkowski space[13]. In euclidean space the imaginary part vanishes. Therefore, the $Z_{B}$ terms are zero.

\section{DIAGRAMMATIC DESCRIPTION}

The contributions to $\mathcal{F}$ have a nice diagrammatic description. Let us represent the scattering amplitudes $\mathcal{M}_{\mathbf{1 2 . . m} ; \mathbf{1 2 . . n}}$ as a vertex with $n$ incoming and $m$ outgoing lines. We construct a diagram with no external lines by linking the lines of vertices. The rules for computing a contribution to the free energy density $\mathcal{F}$ are then the following:

(i) Assign a factor of the scattering amplitude and an energy conserving delta function $2 \pi \delta_{\alpha^{\prime} ; \alpha}^{(E)} \mathcal{M}_{\alpha^{\prime} ; \alpha}$ to each vertex as in Figure 1. 
(ii) Assign a factor $f_{0}(\mathbf{k}) / 2 \omega_{\mathbf{k}}$ to each line as in Figure 1.

(iii) Conserve momentum $\mathbf{k}$ at each vertex and integrate over every unconstrained momentum with $\int d^{(d)} \mathbf{k} /(2 \pi)^{d}$.

(iv) Identify $2 \pi \delta^{(E)}(0)=\beta$.

(v) Divide by the symmetry factor of the diagram, defined as the number of permutations of the internal lines that do not change the topology of the graph, including relative positions. (vi) For fermions determine the overall sign of the diagram: it has an overall factor of $s$ if it arises from an odd permutation of the outgoing state $\left\langle\mathbf{1}^{\prime} \mathbf{2}^{\prime} \ldots\right|$ in the cluster expansion of $\left\langle\mathbf{1}^{\prime} \mathbf{2}^{\prime} \ldots|T| \mathbf{1 2} \ldots\right\rangle$.

(vii) Divide by $-\beta$.

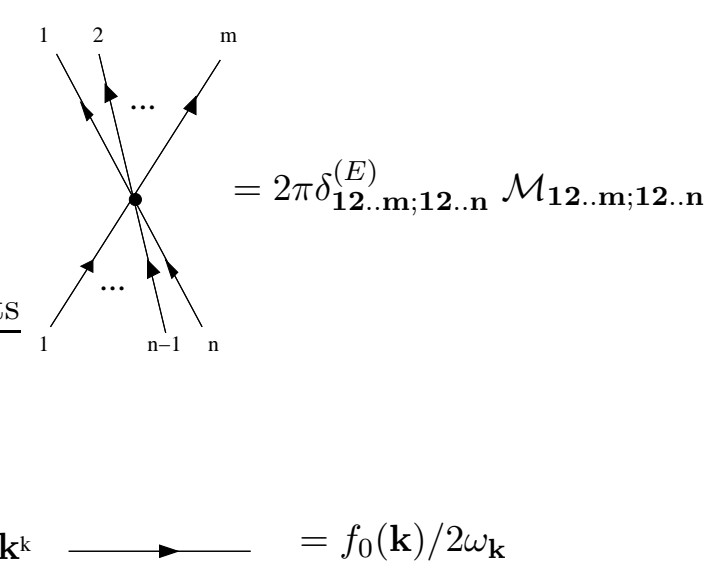

FIG. 1: Diagrammatic ingredients

The terms in eq. (51) are then represented by the diagram in Figure 2. The two terms in eqns. (56, 57) are represented as diagrams in Figure 3.

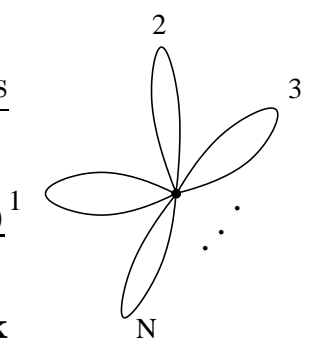

FIG. 2: Diagrammatic representation of terms in eq. (51)

The structure of the "ring" diagrams shown in Figure 4 are especially simple. We compute the sum of such diagrams in order to illustrate the above rules and also since we will need the 


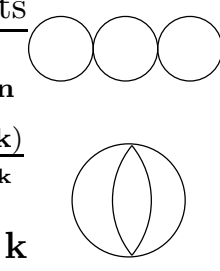

FIG. 3: Diagrammatic representation of the two terms in eq. (56) and (57) respectively.

result in the next section. Let the diagram in Figure 4 be denoted as $\mathcal{F}_{\text {ring }}^{(n)}$. The symmetry factor of this diagram is 2 for $n=1$ and $n$ for $n \geq 2$. For fermions, the overall sign of the diagram is $s^{n+1}$. The sum over $n$ then simply gives a log:

$$
\begin{aligned}
\sum_{n=2}^{\infty} \mathcal{F}_{\text {ring }}^{(n)} & =-\frac{1}{\beta} \int \frac{d^{d} \mathbf{k}}{(2 \pi)^{d}}\left(-\frac{y_{0}(\mathbf{k})}{2}-s \log \left(1-s y_{0}(\mathbf{k})\right)\right) \\
y_{0}(\mathbf{k}) \equiv & =\frac{\beta f_{0}(\mathbf{k})}{2 \omega_{\mathbf{k}}} \int \frac{d^{d} \mathbf{k}^{\prime}}{(2 \pi)^{d}} \mathcal{M}_{\mathbf{1 2 ; 1 2}}\left(\mathbf{k}, \mathbf{k}^{\prime}\right) \frac{f_{0}\left(\mathbf{k}^{\prime}\right)}{2 \omega_{\mathbf{k}^{\prime}}}
\end{aligned}
$$

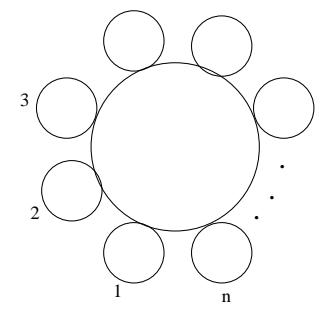

FIG. 4: Ring diagrams.

\section{VI. $\digamma$ AND THE SADDLE POINT EQUATIONS}

We now return to including interactions in the free energy functional $\digamma$ of section II. Let us write

$$
\digamma\left(f, f_{0}\right)=\digamma_{0}\left(f, f_{0}\right)+\digamma_{1}
$$

where $\digamma_{0}$ is given in eq. (11) and we define $U$ as the "potential" which incorporates interactions:

$$
\digamma_{1}=-\frac{1}{\beta} \int \frac{d^{d} \mathbf{k}}{(2 \pi)^{d}} U(\mathbf{k})
$$

It is not too difficult to understand that $\digamma_{1}$ is given by the 2-particle irreducible diagrams of the last section. These are defined as diagrams that cannot become disconnected by the 
cutting of 2 internal lines. For instance, starting from the $N=2$ diagram in Figure 2, it is clear that the ring diagrams can generated by attaching additional loops. The ring diagrams are not 2-particle irreducible and should thus not be included in $\digamma_{1}$. A more detailed argument was given by Lee and Yang $[9]$. We express this explicitly as follows: $\digamma_{1}$ is just $\mathcal{F}_{2-\text { part. irred. }}\left(f_{0}\right)$ with $f_{0}$ replaced by $f$ :

$$
\digamma_{1}(f)=\sum \mathcal{F}_{2-\text { part.irred. }}\left(f_{0} \rightarrow f\right)
$$

This is shown in Figure 4, where it is implicit that the lines have a factor of $f$ rather than $f_{0}$.

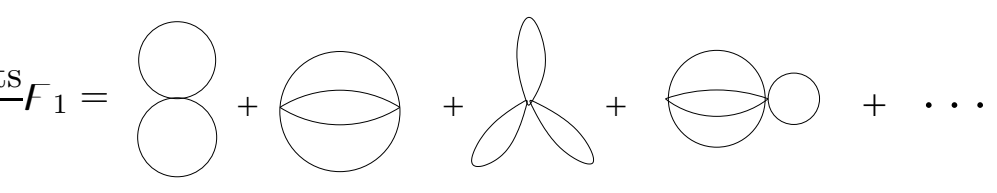

FIG. 5: $\digamma_{1}$ as the sum of 2 particle irreducible diagrams.

Given $\digamma, f$ is determined by the saddle point equation:

$$
\log \left(\frac{1+s f}{f}\right)-\log \left(\frac{1+s f_{0}}{f_{0}}\right)=-\frac{\partial U}{\partial f}
$$

Substituting the solution of (63) back into $\digamma$, it can be written in a variety of ways, depending on how one utilizes the relation (63). One useful way is:

$$
\begin{aligned}
\mathcal{F} & =-\frac{1}{\beta} \int \frac{d^{d} \mathbf{k}}{(2 \pi)^{d}}\left[s \log (1+s f)+\left(1-f \partial_{f}\right) U\right] \\
& =\mathcal{F}_{0}-\frac{1}{\beta} \int \frac{d^{d} \mathbf{k}}{(2 \pi)^{d}}\left[s \log \left(\frac{1+s f}{1+s f_{0}}\right)+\left(1-f \partial_{f}\right) U\right]
\end{aligned}
$$

It is convenient to define a pseudo-energy $\varepsilon$ as the following parameterization of $f$ :

$$
f \equiv \frac{1}{e^{\beta \varepsilon}-s}
$$

Then the saddle point equation and free energy density take the form:

$$
\begin{aligned}
\varepsilon & =\omega-\mu-\frac{1}{\beta} \frac{\partial U}{\partial f} \\
\mathcal{F} & =-\frac{1}{\beta} \int \frac{d^{d} \mathbf{k}}{(2 \pi)^{d}}\left[-s \log \left(1-s e^{-\beta \varepsilon}\right)+\left(1-f \partial_{f}\right) U\right]
\end{aligned}
$$




\section{TWO-BODY APPROXIMATION}

If the gas is not too dense, one expects that 2-particle scattering will give the most important contribution. This is especially true in the non-relativistic case where scattering preserves the number of particles.

Define the following kernel from the 2-particle scattering amplitude:

$$
\mathbf{K}\left(\mathbf{k}, \mathbf{k}^{\prime}\right) \equiv \frac{1}{4 \omega_{\mathbf{k}} \omega_{\mathbf{k}^{\prime}}} \mathcal{M}_{\mathbf{1 2} ; \mathbf{1 2}}\left(\mathbf{k}, \mathbf{k}^{\prime}\right)
$$

and the convolution:

$$
(\mathbf{K} * f)(\mathbf{k}) \equiv \int \frac{d^{d} \mathbf{k}^{\prime}}{(2 \pi)^{d}} \mathbf{K}\left(\mathbf{k}, \mathbf{k}^{\prime}\right) f\left(\mathbf{k}^{\prime}\right)
$$

The 2-particle contribution to $U$ can then be written as:

$$
U(\mathbf{k})=\frac{\beta}{2} f(\mathbf{k})(\mathbf{K} * f)(\mathbf{k})
$$

The saddle point equation and free energy then take the forms:

$$
\begin{gathered}
\log \left(\frac{1+s f}{f}\right)-\log \left(\frac{1+s f_{0}}{f_{0}}\right)=-\beta \mathbf{K} * f \\
\mathcal{F}=-\frac{1}{\beta} \int \frac{d^{d} \mathbf{k}}{(2 \pi)^{d}}\left[s \log (1+s f)+\frac{f}{2} \log \left(\frac{1+s f}{f} \frac{f_{0}}{1+s f_{0}}\right)\right]
\end{gathered}
$$

In terms of the pseudo-energy:

$$
\begin{aligned}
\varepsilon & =\omega-\mu-\mathbf{K} *\left(\frac{1}{e^{\beta \varepsilon}-s}\right) \\
\mathcal{F} & =-\frac{1}{\beta} \int \frac{d^{d} \mathbf{k}}{(2 \pi)^{d}}\left[-s \log \left(1-s e^{-\beta \varepsilon}\right)+\frac{\beta f}{2}(\varepsilon-\omega+\mu)\right]
\end{aligned}
$$

In this two-body approximation, the integral equation (73) resums all diagrams involving two-body scattering. These "foam diagrams" are of the kind shown in Figure 6.

The foam diagrams contain much more than the ring diagrams. As a check, we now show how the ring diagrams are contained in the solution of the integral equation. Let us define $y$ as $\log \left(\frac{1+s f}{1+s f_{0}}\right)=\log (1+y)$ where

$$
y \equiv \frac{f-f_{0}}{1+s f_{0}}
$$

The integral equation (71) can then be expanded in powers of $y$ :

$$
y-\frac{\left(1+2 s f_{0}\right)}{2 f_{0}} y^{2}+\ldots=\beta \mathbf{K} *\left(f_{0}+\left(1+s f_{0}\right) y\right)
$$




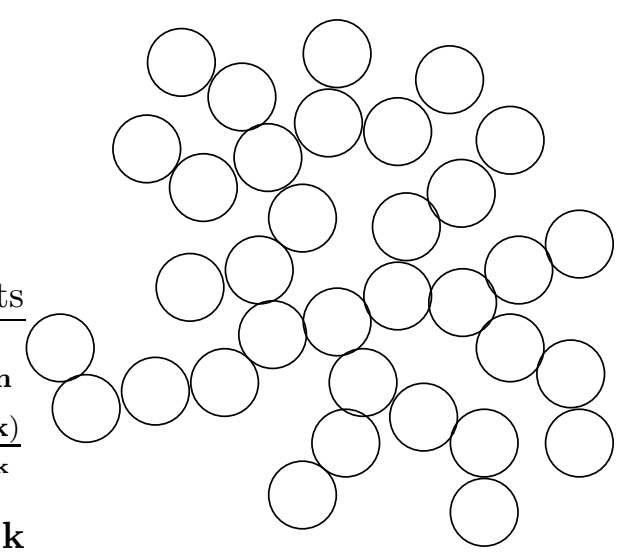

FIG. 6: Foam diagrams.

Now let $y=y_{0}+y_{1}+\ldots$ and plug this into the above equation. Many kinds of terms are generated, but to compare with the ring diagrams we only focus on terms of the type in eq. (59). To lowest orders one finds

$$
y=y_{0}+2 s y_{0}^{2}+\ldots
$$

where $y_{0}$ is defined in eq. (59)). Plugging this lowest order solution into eq. (72) for the free energy one finds

$$
\mathcal{F}-\mathcal{F}_{0}=-\frac{1}{\beta} \int \frac{d^{d} \mathbf{k}}{(2 \pi)^{d}}\left(y_{0}+s y_{0}^{2} / 2+\ldots .\right)
$$

which agrees with the low order expansion of eq. (59).

\section{NON-RELATIVISTIC CASE: HARD-CORE BOSONS}

We have not assumed the underlying theory is Lorentz invariant in the above construction. However, in the definition eq. (34) of the states $\left|\mathbf{k}_{1} \cdots \mathbf{k}_{n}\right\rangle$ we included factors of $\sqrt{2 \omega_{\mathbf{k}}}$ in order that $\underline{\underline{d \mathbf{k}}}$ in eq. (36) is Lorentz invariant. This simplifies the comparision with other approaches for relativistic models, as we did in section IVC for the $\phi^{4}$ theory, since then $\mathcal{M}$ are the conventional scattering amplitudes. 


\section{A. Generalities of the non-relativistic case}

For non-relativistic theories, where $\omega_{\mathbf{k}}=\mathbf{k}^{2} / 2 m$, it is more conventional to normalize the states as

$$
\left|\mathbf{k}_{1} \cdots \mathbf{k}_{N}\right\rangle=a_{\mathbf{k}_{1}}^{\dagger} \cdots a_{\mathbf{k}_{N}}^{\dagger}|0\rangle
$$

The formulas of the previous sections still apply but with the modification:

$$
\underline{\underline{d \mathbf{k}}}=\int \frac{d^{d} \mathbf{k}}{(2 \pi)^{d} 2 \omega_{\mathbf{k}}} \rightarrow \int \frac{d^{d} \mathbf{k}}{(2 \pi)^{d}}
$$

We will keep the definition eq (43) of $\mathcal{M}$ in terms of $\widehat{T}$. The formulas for the two-body approximation in section VII then all apply but now with:

$$
\mathbf{K}\left(\mathbf{k}, \mathbf{k}^{\prime}\right) \equiv \mathcal{M}_{\mathbf{1 2 , 1 2}}\left(\mathbf{k}, \mathbf{k}^{\prime}\right)
$$

The above kernel has dimensions of energy • volume which corresponds to an energy dimension of $1-d$ (up to velocity factors).

\section{B. Hard core boson model}

Consider the two-body potential in position space $\mathbf{x}$ :

$$
V\left(\mathbf{x}, \mathbf{x}^{\prime}\right)=\frac{\gamma}{2} \delta^{(d)}\left(\mathbf{x}-\mathbf{x}^{\prime}\right)
$$

This leads to the second quantized hamiltonian:

$$
H=\int d^{d} \mathbf{x}\left(\frac{1}{2 m} \vec{\nabla} \psi^{\dagger} \cdot \vec{\nabla} \psi+\frac{\gamma}{4} \psi^{\dagger} \psi^{\dagger} \psi \psi\right)
$$

To lowest order $T=H_{1}$ and

$$
\left\langle\alpha^{\prime}\left|H_{1}\right| \alpha\right\rangle=-(2 \pi)^{d} \delta^{(d)}\left(\mathbf{k}_{\alpha}-\mathbf{k}_{\alpha^{\prime}}\right) \mathcal{M}_{\alpha^{\prime} ; \alpha}
$$

Expanding the field in terms of annihilation operators:

$$
\psi(\mathbf{x})=\int \frac{d^{d} \mathbf{k}}{(2 \pi)^{d}} e^{i \mathbf{k} \cdot \mathbf{x}} a_{\mathbf{k}}
$$

then to lowest order one finds

$$
M_{12 ; 12}=-\gamma=\mathbf{K}
$$


The coupling constant $\gamma$ has units of energy $\times$ volume. It can be expressed in terms of a physical scattering length $a$ as follows. To first order in perturbation theory the differential cross-section in the center of mass is:

$$
\frac{d \sigma}{d \Omega}=\frac{m^{2} \gamma^{2}}{4(2 \pi)^{d-1}} k^{d-3}
$$

where $k$ is the magnitude of $\mathbf{k}$ for one of the incoming particles. Since a cross section has dimensions of length ${ }^{d-1}$, we define $a$ such that the cross section is $a^{d-1}$ when the wavelength of the particle is $2 \pi / a$ :

$$
\left.\frac{d \sigma}{d \Omega}\right|_{k \sim 2 \pi / a} \sim a^{d-1}
$$

This leads us to make the definition:

$$
\frac{\gamma}{(2 \pi)^{d / 2}} \equiv \frac{a^{d-2}}{m}
$$

\section{Lowest order solution}

It is interesting to carry out our analysis for arbitrary spacial dimension $d>0$. Integrals over momenta can be traded for integrals over $\omega$ :

$$
\int \frac{d^{d} \mathbf{k}}{(2 \pi)^{d}}=\left(\frac{m}{2 \pi}\right)^{d / 2} \frac{1}{\Gamma(d / 2)} \int_{0}^{\infty} d \omega \omega^{(d-2) / 2}
$$

For a constant kernel $\mathbf{K}=-\gamma$, and $d>0$, the solution to the integral equation eq. (73) takes the simple form:

$$
\varepsilon(\mathbf{k})=\omega_{\mathbf{k}}-\mu+T \delta
$$

where $\delta$ is independent of $\mathbf{k}$ and satisfies the equation [29]

$$
\delta=h \operatorname{Li}_{d / 2}\left(z_{\mu} z_{\delta}\right)
$$

where we have defined the fugacities

$$
z_{\mu} \equiv e^{\beta \mu}, \quad z_{\delta}=e^{-\delta}
$$

and a renormalized thermal coupling $h$ and thermal wavelength $\lambda_{T}$ :

$$
h \equiv\left(\frac{a}{\lambda_{T}}\right)^{d-2}, \quad \lambda_{T} \equiv \frac{1}{\sqrt{m T}}
$$


The function $\operatorname{Li}_{\nu}(z)$ is the standard polylogarithm, defined as the appropriate analytic continuation of

$$
\operatorname{Li}_{\nu}(z)=\sum_{n=1}^{\infty} \frac{z^{n}}{n^{\nu}}
$$

The equation (92) is thus seen as non-trivial, transcendental equation that determines $\delta$ as a function of $\mu, T$, and the coupling $h$. Given the solution $\delta(\mu)$ of this equation, using eq. (74) the density can be expressed as

$$
n(\mu)=\frac{1}{\left(2 \pi \lambda_{T}^{2}\right)^{d / 2}} \operatorname{Li}_{d / 2}\left(z_{\mu} z_{\delta}\right)=\frac{T \delta}{\gamma}
$$

For $d>0$ one can integrate by parts and obtain the following expressions for the free energy

$$
\mathcal{F}=-\frac{T}{\left(2 \pi \lambda_{T}^{2}\right)^{d / 2}}\left(\operatorname{Li}_{(d+2) / 2}\left(z_{\mu} z_{\delta}\right)+\frac{\delta}{2} \operatorname{Li}_{d / 2}\left(z_{\mu} z_{\delta}\right)\right)
$$

The case of $2 d$ is interesting since the eq. (92) then becomes algebraic due to $\operatorname{Li}_{1}(z)=$ $-\log (1-z)$. These formulas will be applied to Bose-Einstein condensation, and other problems, in [21].

\section{CONCLUDING REMARKS}

In this paper we have mainly focused on developing the formalism in a general way for both relativistic and non-relativistic theories. In the two-body approximation, the main result is summarized in the two formulas eqs. (73) plement: one computes, or measures, the 2-body forward-scattering kernel $\mathbf{K}$ at zero temperature, solves the integral equation (73), and the free energy is given in terms of this solution in eq. (74). For non-constant kernels, one will probably need to solve the equations numerically.

A formalism that efficiently disentangles zero temperature dynamics and quantum statistical sums potentially has many applications, and we discuss some of them in these concluding remarks.

Our formalism is especially well-suited for studies of the effects of interactions in BoseEinstein condensation, since there the filling fractions play a central role. We have already

obtained some results on this problem and will publish them elsewhere[21]. The results presented there also give new insights on the Riemann hypothesis. 
For high-energy particle physics, our formalism has the following potential advantages over the Matsubara approach. The necessity to renormalize ultra-violet divergences is essentially a zero temperature problem, however the Matsubara approach again entangles the zero-temperature renormalization with the quantum statistical mechanics and the issue of scheme-dependent results can sometimes be a problem. In our formalism, any need for renormalization is carried out at zero temperature and the S-matrix is expressed in terms of physical quantities at zero temperature, such as particle masses, etc. Our integral equation could also shed some light on the infra-red problems that are common in the Matsubara approach and also require special resummations [1, 2, 17, 18, 19, 20]. We emphasize that since the diagrams in this paper have no relation to finite temperature Feynman diagrams, our type of resummation is entirely different than the resummations carried out in these other works. Some aspects of the relativistic case will be published in [22]. Our formalism may help to study the known results on the free energy of strongly coupled supersymmetric gauge theories[24].

Our formalism may also be well suited to studies of the quark-gluon plasma [23] currently being studied at RHIC, since many of the hadronic cross-sections are known.

\section{ACKNOWLEDGMENTS}

I would like to thank G. Mussardo and H. Thacker for discussions and for drawing my attention to the work [6].

\section{APPENDIX A}

In this appendix we complete the steps leading to the result (25) obtained in [6]. Starting from eq. (22), the next step is to try and use the relation $G_{0}-G_{0}^{\dagger}=-2 \pi i \delta\left(E-H_{0}\right)$ so that one can integrate over $E$. One way to do this uses the operators $\Omega$ :

$$
\begin{gathered}
\Omega(E) \equiv G G_{0}^{-1}=1+G_{0} T \\
\Omega^{-1}(E)=G_{0} G^{-1}=1-G_{0} H_{1}
\end{gathered}
$$

As we show below, $\Omega$ is the operator that relates free-particle states to the in-states of scattering theory. Introduce the notation $X \stackrel{\leftrightarrow}{\partial} Y \equiv X(\partial Y)-(\partial X) Y$. Then, using $\partial_{E} G_{0}=$ 
$-G_{0}^{2}$ and $H_{1} \Omega=T$ one can readily show that

$$
\Omega^{-1} \stackrel{\leftrightarrow}{\partial}_{E} \Omega=-2 G_{0}^{2} T
$$

In order to deal with the complex conjugation needed in eq. (22), for any operator $X(E)$ define $X^{*}(E)$ as simply $X(E)$ but with $i \varepsilon$ replaced by $-i \varepsilon$. This definition gives $G^{*}(E)=G(E)^{\dagger}, \Omega^{*}(E)=1+G^{\dagger} H_{1}$, and $\left(\Omega^{-1}\right)^{*}=1-G_{0}^{\dagger} H_{1}$. One then has

$$
\left(\Omega^{-1}\right)^{*} \stackrel{\leftrightarrow}{\partial}_{E} \Omega^{*}=-2\left(G_{0}^{\dagger}\right)^{2} T^{\dagger}
$$

The last step is to define

$$
S(E)=\left(\Omega^{-1}\right)^{*} \Omega(E)
$$

Using eq. (22, 99), and the cyclicity of the trace, one obtains

$$
Z=Z_{0}+\frac{1}{4 \pi i} \int_{0}^{\infty} d E e^{-\beta E} \operatorname{Tr}\left(\mathrm{S}^{-1} \stackrel{\leftrightarrow}{\partial}_{\mathrm{E}} \mathrm{S}\right)
$$

We now demonstrate that the so-called on-shell matrix elements of the S-operator are the usual S-matrix. Consider first the operator $\Omega$. Inserting a complete set of states $\left|\alpha^{\prime}\right\rangle$ one finds:

$$
\Omega\left(E_{\alpha}\right)|\alpha\rangle=|\alpha\rangle+\sum_{\alpha^{\prime}} \frac{1}{E_{\alpha}-E_{\alpha^{\prime}}+i \varepsilon} T_{\alpha^{\prime} \alpha}\left|\alpha^{\prime}\right\rangle
$$

where

$$
T_{\alpha^{\prime} \alpha}=\left\langle\alpha^{\prime}|T(E)| \alpha\right\rangle, \quad \text { iff } E=E_{\alpha}
$$

The condition $E=E_{\alpha}$ is the on-shell condition. Eq. (103) are the Lippmann-Schwinger equations:

$$
|\alpha\rangle_{\text {in }}=\Omega\left(E_{\alpha}\right)|\alpha\rangle
$$

which arise from attempting to solve $H|\alpha\rangle_{\text {in }}=E_{\alpha}|\alpha\rangle_{\text {in }}$ for the in-states (which also have energy $\left.E_{\alpha}\right)$.

Turning now to the S-operator, using identities given above, one can easily establish

$$
\begin{aligned}
S(E) & =1-2 \pi i \delta\left(E-H_{0}\right) T(E) \\
S^{-1}(E) & =1+2 \pi i \delta\left(E-H_{0}\right) T^{\dagger}(E)
\end{aligned}
$$

The latter implies

$$
\left\langle\alpha^{\prime}|S(E)| \alpha\right\rangle=\left\langle\alpha^{\prime} \mid \alpha\right\rangle-2 \pi i \delta\left(E_{\alpha}-E_{\alpha^{\prime}}\right) T_{\alpha^{\prime} \alpha}, \quad \text { iff } \quad E=E_{\alpha}
$$


The equation (102) together with the identification (106) of the S-matrix is the main result obtained in [6].

[1] J. I. Kapusta, Finite Temperature Field Theory, Cambridge, 1989

[2] M. Le Bellac, Thermal Field Theory, Cambridge, 1996.

[3] L. Dolan and R. Jackiw, Phys. Rev. D9 (1974) 3320.

[4] C. N. Yang and C. P. Yang, Jour. Math. Phys. 10, (1969) 1115.

[5] Al. Zamolodchikov, Nucl. Phys. B342 (1990) 695.

[6] R. Dashen, S.-K. Ma and H. J. Bernstein, Phys. Rev. 187 (1969) 345.

[7] H. B. Thacker, Phys. Rev. D16 (1977) 2515.

[8] R. Dashen and S.-K. Ma, J. Math. Phys. 12 (1971) 689.

[9] T. D. Lee and C. N. Yang, Phys. Rev. 113 (1959) 1165; Phys. Rev. 117 (1960) 22.

[10] L. D. Landau and E. M. Lifshitz, Statistical Physics, Pergamon Press (1980).

[11] K. Gottfried and T.-M. Yan, Quantum Mechanics: Fundamentals, Spring-Verlag, 2003.

[12] S. Weinberg, The Quantum Theory of Fields I, Cambridge University Press 1995.

[13] M. E. Peskin and D. V. Schroeder, An Introduction to Quantum Field Theory, Addison-Wesley 1995.

[14] A. LeClair, F. Lesage, S. Sachdev, and H. Saleur, Nucl.Phys. B482 (1996) 579 cond-mat/9606104.

[15] A. LeClair and G. Mussardo, Nucl.Phys.B552 (1999) 624.

[16] A. I. Bugrij and V. N. Shadura, arXiv:hep-th/9510232.

[17] R. R. Parwani, Phys.Rev. D45 (1992) 4695; Erratum-ibid. D48 (1993) 5965, hep-ph/9204216.

[18] E. Braaten and R. D. Pisarski, Nucl. Phys. B337 (1990) 569; Nucl. Phys. B339 (1990) 310; J. Frenkel and J. C. Taylor, Nucl. Phys. B334 (1990) 199.

[19] I. T. Drummond, R. R. Horgan, and P. V. Landshoff, Nucl.Phys. B524 (1998) 579, hep-ph/9708426

[20] J.-P. Blaizot, E. Iancu, and A. Rebhan, Phys.Rev. D63 (2001) 065003, hep-ph/0005003.

[21] A. LeClair, Interacting Bose and Fermi gases in low dimensions and the Riemann hypothesis, to appear.

[22] A. LeClair, Pressure of relativistic quantum gases from the S-matrix,, to appear. 
[23] M. G. Alford, K. Rajagopal, and F. Wilczek, Phys. Lett. B422 (1998) 247.

[24] S. S. Gubser, I. R. Klebanov, and A. A. Tseytlin, Nucl. Phys. B534 (1988) 202, hep-th/9805156.

[25] In this $2 D$ context, the central charge $c$ is the coefficient of the conformal free energy $\mathcal{F}=$ $-c \pi T^{2} / 6$ and $c$ is the same $c$ as appears in the Virasoro algebra.

[26] To our knowledge, a complete derivation of the thermodynamic Bethe ansatz equations from this formalism is not known. Some steps in this direction were taken in [7].

[27] $(A-B)^{-1}=A^{-1}\left(1+B(A-B)^{-1}\right)=\left(1+(A-B)^{-1} B\right) A^{-1}$.

[28] Another form of eq. (25) is based on the identity $\operatorname{Tr}^{-1} \stackrel{\leftrightarrow}{\partial} \mathrm{E} S=2 \mathrm{i} I m \operatorname{Tr} \partial_{\mathrm{E}} \log \mathrm{S}$, where we have used the on-shell relation $S^{-1}=S^{\dagger}$. Though this form may be useful to compare with the TBA, we do not use it in this paper.

[29] We have used $\int_{0}^{\infty} d x x^{\nu-1} /\left(e^{x} / z-1\right)=\Gamma(\nu) \operatorname{Li}_{\nu}(z)$ for $\operatorname{Re}(\nu)>0$. 